Research article

Open Access

\title{
Melanocortin peptides inhibit urate crystal-induced activation of phagocytic cells
}

\author{
Franco Capsoni ${ }^{1}$, Anna Maria Ongari ${ }^{1}$, Eva Reali² and Anna Catania ${ }^{3}$
}

\author{
${ }^{1}$ Rheumatology Unit, Istituto Ortopedico Galeazzi IRCCS (Istituto Di Ricovero e Cura a Carattere Scientifico), University of Milan, Via Riccardo \\ Galeazzi 4, 20161 Milan, Italy \\ 2INGM-National Institute of Molecular Genetics, Fondazione IRCCS Ospedale Maggiore Policlinico, Mangiagalli e Regina Elena, Via Francesco \\ Sforza 28, 20122 Milan, Italy \\ ${ }^{3}$ Center for Preclinical Investigation, Fondazione IRCCS Ospedale Maggiore Policlinico, Mangiagalli e Regina Elena, Via Francesco Sforza 28, 20122 \\ Milan, Italy \\ Corresponding author: Franco Capsoni, franco.capsoni@unimi.it
}

Received: 22 Apr 2009 Revisions requested: 18 May 2009 Revisions received: 18 Sep 2009 Accepted: 8 Oct 2009 Published: 8 Oct 2009

Arthritis Research \& Therapy 2009, 11:R151 (doi:10.1186/ar2827)

This article is online at: http://arthritis-research.com/content/11/5/R151

(C) 2009 Capsoni et al.; licensee BioMed Central Ltd.

This is an open access article distributed under the terms of the Creative Commons Attribution License (http://creativecommons.org/licenses/by/2.0), which permits unrestricted use, distribution, and reproduction in any medium, provided the original work is properly cited.

\begin{abstract}
Introduction The melanocortin peptides have marked antiinflammatory potential, primarily through inhibition of proinflammatory cytokine production and action on phagocytic cell functions. Gout is an acute form of arthritis caused by the deposition of urate crystals, in which phagocytic cells and cytokines play a major pathogenic role. We examined whether alpha-melanocyte-stimulating hormone $(\alpha-\mathrm{MSH})$ and its synthetic derivative $(\mathrm{CKPV})_{2}$ influence urate crystal-induced monocyte (Mo) activation and neutrophil responses in vitro.

Methods Purified Mos were stimulated with monosodium urate (MSU) crystals in the presence or absence of melanocortin peptides. The supernatants were tested for their ability to induce neutrophil activation in terms of chemotaxis, production of reactive oxygen intermediates (ROIs), and membrane expression of CD11b, Toll-like receptor-2 (TLR2) and TLR4. The proinflammatory cytokines interleukin (IL)-1 $\beta$, IL-8, and tumor necrosis factor-alpha (TNF- $\alpha$ ) and caspase- 1 were determined in the cell-free supernatants. In parallel experiments, purified neutrophils were preincubated overnight with or without melanocortin peptides before the functional assays.
\end{abstract}

Results The supernatants from MSU crystal-stimulated Mos exerted chemoattractant and priming activity on neutrophils, estimated as ROI production and CD11b membrane expression. The supernatants of Mos stimulated with MSU in the presence of melanocortin peptides had less chemoattractant activity for neutrophils and less ability to prime neutrophils for CD11b membrane expression and oxidative burst. MSU crystalstimulated Mos produced significant levels of IL-1 $\beta$, IL-8, TNF- $\alpha$, and caspase-1. The concentrations of proinflammatory cytokines, but not of caspase-1, were reduced in the supernatants from Mos stimulated by MSU crystals in the presence of melanocortin peptides. Overnight incubation of neutrophils with the peptides significantly inhibited their ability to migrate toward chemotactic supernatants and their capacity to be primed in terms of $\mathrm{ROI}$ production.

Conclusions $\alpha-\mathrm{MSH}$ and (CKPV) ${ }_{2}$ have a dual effect on MSU crystal-induced inflammation, inhibiting the Mos' ability to produce neutrophil chemoattractants and activating compounds and preventing the neutrophil responses to these proinflammatory substances. These findings reinforce previous observations on the potential role of $\alpha-\mathrm{MSH}$ and related peptides as a new class of drugs for treatment of inflammatory arthritis.

$\alpha-\mathrm{MSH}$ : alpha-melanocyte-stimulating hormone; BSA: bovine serum albumin; CL: chemiluminescence; ELISA: enzyme-linked immunosorbent assay; FBS: fetal bovine serum; FMLP: $N$-formyl-methionyl-leucyl-phenylalanine; IL: interleukin; LPS: lipopolysaccharide; mAb: monoclonal antibody; Mo: monocyte; MSU: monosodium urate; NF-кB: nuclear factor-kappa-B; PBS: phosphate-buffered saline; ROI: reactive oxygen intermediate; SMM: supernatant from monosodium urate-stimulated monocytes; TLR: Toll-like receptor; TNF- $\alpha$ : tumor necrosis factor-alpha. 


\section{Introduction}

Alpha-melanocyte-stimulating hormone ( $\alpha-\mathrm{MSH})$ is an endogenous tridecapeptide with multiple effects on host cells. The synthetic peptide inhibits inflammatory responses in experimental models of acute and chronic disorders, including bowel diseases, allergy, adjuvant arthritis, and sepsis [1-4]. $\alpha$ $\mathrm{MSH}$ interacts with host cells through recognition of specific melanocortin receptors (MCRs 1 to 5). Its anti-inflammatory action depends primarily on inhibition of cytokine production by target cells. This is achieved by preventing the activation of nuclear transcription factor-kappa-B (NF- $\kappa \mathrm{B}$ ) (reviewed in [4]). Several leukocyte functions, including reactive oxygen intermediate (ROI) generation and release of proteolytic enzymes, are also influenced by $\alpha-\mathrm{MSH}$. Nitric oxide production and the expression of adhesion molecules are likewise inhibited in both neutrophils and monocytes (Mos) [5,6]. $\alpha-\mathrm{MSH}$ inhibits human neutrophil migration and several other interleukin-8 (IL8)-induced biological responses [7-9]; inhibition of antigenstimulated lymphocyte proliferation has been reported also [10]. The significant role of $\alpha-\mathrm{MSH}$ and related peptides in immune/inflammatory responses and their ability to prevent inflammation-mediated tissue injury suggest these molecules as a potential new class of anti-inflammatory drugs. However, with a view to this use, cost-effective stable analogs need to be developed.

Previous observations indicated that the anti-inflammatory message sequence of $\alpha-\mathrm{MSH}$ [1-13] resides in the C-terminal tripeptide Lys-Pro-Val (MSH 11-13 or KPV) [11]. A dimer obtained by inserting a Cys-Cys linker between two units of $\mathrm{KPV},(\mathrm{CKPV})_{2}$, inhibited tumor necrosis factor-alpha (TNF- $\alpha$ ) production by lipopolysaccharide (LPS)-stimulated human leukocytes with potency similar to the stable $\alpha-\mathrm{MSH}$ analog [Nle4-dPhe7]- $\alpha-\mathrm{MSH}$ (NDP- $\alpha-\mathrm{MSH})$ and effectiveness greater than KPV. Effectiveness was similar in vivo: (CKPV) markedly inhibited circulating TNF- $\alpha$ after intravenous injection of LPS and significantly reduced TNF- $\alpha$ and $\mathrm{NO}_{2}-$ concentrations in plasma and in the peritoneal cavity in a rat model of LPS-induced peritonitis [12].

We recently reported that $(\mathrm{CKPV})_{2}$ in vitro reduced several human neutrophil activities important for the host inflammatory response. These inhibitory effects included cell migration, ROI production, proinflammatory cytokine synthesis and secretion, and expression of adhesion molecules [9].

Gout is an acute form of arthritis causing substantial inflammation and involving tissue deposition of monosodium urate (MSU) crystals. Both Mos and neutrophils are important in pathogenesis. Recent observations suggest that MSU crystals act as danger signals with the ability to activate caspase1 in an NALP-3 inflammosome-dependent manner, with production and release of active IL-1 $\beta$ [13]. In this model, the second phase of the inflammatory response is driven by the IL-1 $\beta$ / IL-1R pathway with activation of an MyD88-dependent signal- ing pathway and NF- $\mathrm{KB}$ activation followed by production of inflammatory mediators that elicit neutrophil recruitment into the joints, characteristic of the acute gouty inflammation [14].

The importance of Mos and neutrophils in the pathogenesis of acute gout suggests that $\alpha-\mathrm{MSH}$ and related peptides control MSU-induced inflammation. This was suggested by Getting and colleagues $[15,16]$, who showed that melanocortins had protective action in a rat model of gouty arthritis. Therefore, we examined whether $\alpha-\mathrm{MSH}$ and the synthetic melanocortin $(\mathrm{CKPV})_{2}$ influenced MSU crystal-induced human Mo activation and human neutrophil responses in vitro.

\section{Materials and methods Reagents}

The peptides (CKPV) ${ }_{2}$ and $\alpha-\mathrm{MSH}$ [1-13], N-acetylated and C-amidated, were kindly provided by Paolo Grieco, Department of Pharmaceutical and Toxicological Chemistry, University of Naples Federico II, Italy. Lymphoprep gradient (density $1.077 \mathrm{~g} / \mathrm{mL}$ ) and Nycoprep 1.068 gradient were purchased from Axis-Shield (Oslo, Norway). Extra-low endotoxin fetal bovine serum (FBS) was obtained from HyClone (Logan, UT, USA). Hanks' balanced salt solution, RPMI 1640, penicillin, streptomycin, glutamine, bovine serum albumin (BSA), $\mathrm{N}$ formyl-methionyl-leucyl-phenylalanine (FMLP), luminol (5amino-2,3-dihydro-1,4-phthalazinedione), and polymyxin B sulphate were from Sigma-Aldrich (St. Louis, MO, USA). Micropore filters were from Millipore Corporation (Bedford, MA, USA). The caspase-1 inhibitor z-YVAD-fmk was from Alexis Biochemicals (Farmingdale, NY, USA). The phycoerythrinated anti-Toll-like receptor-2 (TLR2), anti-TLR4, and anti-CD11b monoclonal antibodies (mAbs) were from eBioscence, Inc. (San Diego, CA, USA). Irrelevant class-matched mAbs used as controls for nonspecific binding were from Becton, Dickinson and Company (Franklin Lakes, NJ, USA). The mAbs antiIL-1 $\beta$ and anti-IL-8 and the specific enzyme-linked immunosorbent assay (ELISA) for IL-1 $\beta, \mathrm{IL}-8, \mathrm{TNF}-\alpha$, and caspase- 1 were from R\&D Systems (Abingdon, UK). MSU crystals were prepared according to the method described by Murakami and colleagues [17]. The crystals were sterilized by heating at $180^{\circ} \mathrm{C}$ for 2 hours, were resuspended in phosphate-buffered saline (PBS) at a concentration of $10 \mathrm{mg} / \mathrm{mL}$, and were verified free of endotoxin by the Limulus amoebocyte cell lysate assay (Sigma-Aldrich).

\section{Neutrophils and monocyte isolation and culture}

Peripheral blood neutrophils were obtained by density gradient centrifugation (Lymphoprep) [18]. The purified cell population consisted of greater than $95 \%$ pure, viable neutrophils, assessed on the basis of morphology and Trypan blue exclusion. In some experiments, purified neutrophils, at the appropriate density $\left(2.5 \times 10^{6} / \mathrm{mL}\right)$, were incubated in polypropilene tissue culture tubes (Corning Incorporated, Corning, NY, USA) at $37^{\circ} \mathrm{C}$ in a humidified atmosphere of $5 \% \mathrm{CO}_{2}$ for 18 hours in complete medium alone (see below), $\alpha-\mathrm{MSH} 10^{-6} \mathrm{M}$, 
or (CKPV $)_{2} 10^{-6} \mathrm{M}$. At the end of incubation, neutrophils were used in the functional assays as described below. Viability of cells was always more than $95 \%$.

Peripheral blood Mos were purified on a Nycoprep 1.068 gradient (as described in detail by Boyum [19]), which routinely yielded $85 \%$ to $90 \%$ Mo as assessed by Wright staining, nonspecific esterase staining, and (in some cases) immunofluorescent staining for CD14; viability was more than $95 \%$ by Trypan blue exclusion. Freshly isolated Mos were resuspended in RPMI 1640 containing 10\% heat-inactivated FBS (30 minutes at $56^{\circ} \mathrm{C}$ ), $100 \mathrm{U} / \mathrm{mL}$ penicillin, $100 \mu \mathrm{g} / \mathrm{mL}$ streptomycin, and $2 \mathrm{mM}$ glutamine (complete medium) at $1 \times 10^{6}$ Mos $/ \mathrm{mL}$. The Mo suspensions were incubated in polystyrene tissue culture flasks (cell growth area $25 \mathrm{~cm}^{2}$; Corning Incorporated) at $37^{\circ} \mathrm{C}$ in a $95 \%$ air- $5 \% \mathrm{CO}_{2}$ humidified atmosphere for 24 hours in complete medium with or without MSU crystals $(1 \mathrm{mg} / \mathrm{mL})$ and with or without the indicated concentrations of $\alpha-\mathrm{MSH}$ or $(\mathrm{CKPV})_{2}$.

To check for artifacts of trace endotoxin contamination of MSU crystals, the cells were incubated with MSU in the presence of polymyxin B sulphate $(10 \mu \mathrm{g} / \mathrm{mL})$ in some experiments. In three separate experiments, the caspase-1 inhibitor z-YVADfmk was added to the cultured cells at a final concentration of $10 \mu \mathrm{M}$. The supernatants of cultured Mos were collected by centrifugation $(1,200 \mathrm{~g}$ for 15 minutes), were filtered through a $0.22-\mu \mathrm{m}$ filter (Millipore Corporation), and were stored at $80^{\circ} \mathrm{C}$ until tested for their capacity to activate neutrophils and for their content of IL- $1 \beta$, IL-8, TNF- $\alpha$, and caspase-1. The concentration of MSU crystals we used $(1 \mathrm{mg} / \mathrm{mL})$ had been found to be optimal beforehand on a limited dose-response curve $(0.1,1.0$, and $10.0 \mathrm{mg} / \mathrm{mL}$; data not shown) and was similar to that used by others in similar experimental conditions $[16,20,21]$. The viability of cultured Mos, determined by Trypan blue exclusion, always exceeded $90 \%$.

\section{Chemotaxis}

Neutrophil chemotaxis was examined using a modified Boyden chamber assay with blind-well chambers and 3- $\mu \mathrm{m}$ micropore filters [22]. Briefly, $200 \mu \mathrm{L}$ of the cell suspension containing $3.75 \times 10^{6}$ neutrophils $/ \mathrm{mL}$ in RPMl $1640+0.4 \%$ BSA was layered on top of the filter, and the lower compartment was filled with $200 \mu \mathrm{L}$ of the supernatants from MSU-stimulated Mos (SMMs) or the chemotactic factor FMLP $\left(10^{-8} \mathrm{M}\right.$ final concentration). After 120 minutes of incubation at $37^{\circ} \mathrm{C}$ in a humidified atmosphere with $5 \% \mathrm{CO}_{2}$, the filters were fixed with ethanol and stained with hematoxylin-eosin. The chemotactic response was determined by counting the number of cells per high-power field which had migrated through the entire thickness of the filter; duplicate chambers were used in each experiment, and five fields were examined in each filter. In all cases, the person scoring the assay was blind to the experimental groupings. In some experiments, anti-IL-1 $\beta$ (1 $\mu \mathrm{g} / \mathrm{mL}$ final concentration) or anti-lL-8 $(4 \mu \mathrm{g} / \mathrm{mL}$ final concen- tration) or the two mAbs together were added to the SMMs before as chemoattractants.

\section{Chemiluminescence}

Luminol-amplified chemiluminescence $(\mathrm{CL})$ was used to examine the phagocyte production of ROls in response to MSU crystals. To measure $\mathrm{CL}, 2.5 \times 10^{5}$ neutrophils were mixed in 3-mL polystyrene vials with $5 \times 10^{-5} \mathrm{M}$ luminol in a final volume of $700 \mu \mathrm{L}$. The vials were placed in a Luminometer 1251 (LKB Wallac, Turku, Finland) and allowed to equilibrate in the dark for 5 minutes at $37^{\circ} \mathrm{C}$, with intermittent shaking, and then the background light output was recorded in millivolts. MSU crystals (3 $\mathrm{mg} / \mathrm{mL}$ final concentration) [23] were added using an appropriate dispenser (1291; LKB Wallac), and the CL was recorded continuously. The background counts were subtracted from the values obtained after neutrophil stimulation. To check the effect of the SMMs on CL production, neutrophils were preincubated with undiluted supernatants for 30 minutes at $37^{\circ} \mathrm{C}$ before the $\mathrm{CL}$ assay.

\section{Surface expression of CD11b, TLR2, and TLR4}

Flow cytometry of purified neutrophils was used to determine the membrane expression of CD11b, TLR2, and TLR4. Phycoerythrin-conjugated mAbs were added to $100 \mu \mathrm{L}$ of a purified cell suspension $\left(2 \times 10^{5}\right.$ cells in PBS containing $0.1 \% \mathrm{NaN}_{3}$ and $10 \%$ human $A B$ serum). The staining reactions were developed at $4^{\circ} \mathrm{C}$ for 30 minutes. After washing, the cells were analyzed by flow cytometry (Becton Dickinson FACS II; Becton, Dickinson and Company). A relative measure of antigen expression was obtained using the mean fluorescence intensity, converted from a log to a linear scale, after subtracting the cells' self-fluorescence and the fluorescence of cells incubated with irrelevant isotype control mAbs.

\section{Production of cytokines in vitro}

IL-1 $\beta$, IL-8, TNF- $\alpha$, and caspase-1 protein concentrations were determined in the cell-free supernatants using specific ELISA in accordance with the procedures indicated by the manufacturer (R\&D Systems).

\section{Statistical analysis}

The data are expressed as mean \pm standard error of the mean. Statistical analysis was done using the Student $t$ test for unpaired or paired data, as appropriate. A probability of less than 0.05 was considered significant.

\section{Results \\ Monosodium urate-stimulated monocytes produce neutrophil-activating mediators}

Mos were incubated with MSU for 24 hours with or without $\alpha$ $\mathrm{MSH}$ or $(\mathrm{CKPV})_{2}$; the cell-free supernatants were then tested for their ability to induce neutrophil responses. The SMMs showed significant chemotactic activity for neutrophils; this was not due to contaminating LPS since the activity was no different in SMMs from Mos cultured with polymyxin B sul- 


\section{Figure 1}

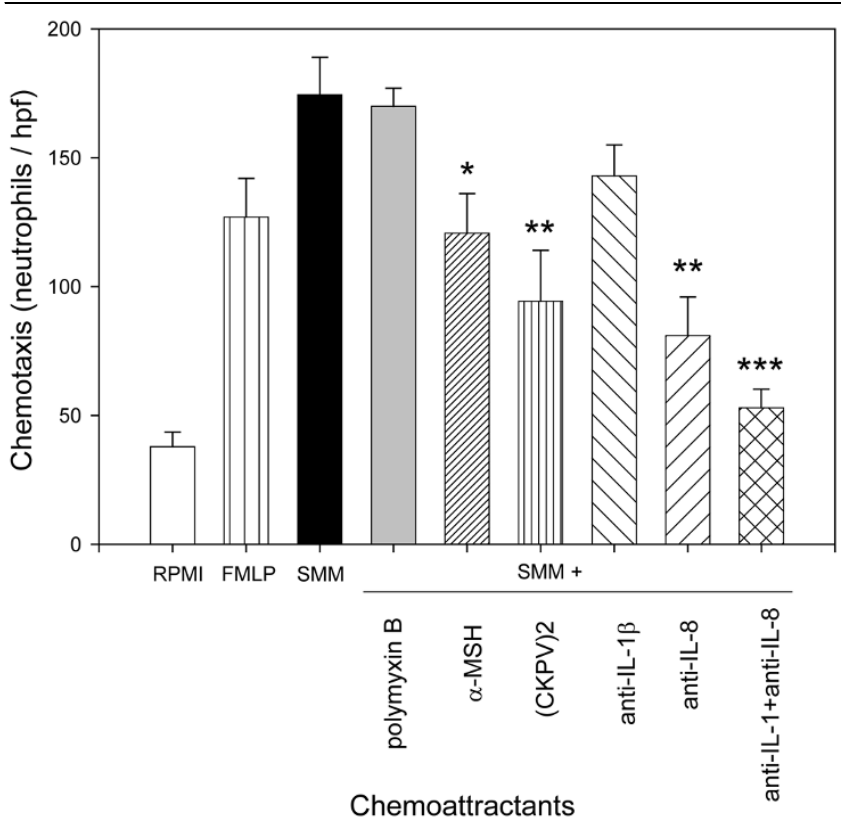

Chemotactic properties of supernatants from monosodium urate (MSU) crystal-stimulated monocytes (SMMs). Monocytes were incubated for 18 hours with MSU crystals $(1 \mathrm{mg} / \mathrm{mL})$ with or without alpha-melanocyte-stimulating hormone $(\alpha-\mathrm{MSH})\left(10^{-6} \mathrm{M}\right)$ or $(\mathrm{CKPV})_{2}\left(10^{-6} \mathrm{M}\right)$ or polymyxin $B(10 \mu \mathrm{g} / \mathrm{mL})$. The cell-free supernatants from 10 separate experiments (two with polymyxin B) were used as chemoattractants for purified human neutrophils. In three separate experiments, SMMs were preincubated (15 minutes at room temperature) with anti-interleukin-1 (anti-IL-1) or anti-IL-8 monoclonal antibodies or both before use as chemoattractants. The chemotactic activity of the standard chemoattractant $N$-formyl-methionyl-leucyl-phenylalanine (FMLP) $\left(10^{-8} \mathrm{M}\right)$ is shown. Results are expressed as number of neutrophils per high-power field (hpf). Bars denote mean \pm standard error of the mean. ${ }^{\star} P<0.05 ;{ }^{\star \star} P<$ $0.01 ;{ }^{* \star \star} P<0.005$ versus SMMs.

phate (Figure 1). The chemotactic activity of SMMs was similar to or even greater than the standard chemoattractant FMLP (Figure 1). MSU crystals induced CL production by neutrophils. These SMMs also had a priming effect on neutrophils: when neutrophils were preincubated with the SMMs, they showed an enhanced respiratory burst in response to a challenge with MSU (Figure 2). In addition, neutrophils pretreated with the SMMs showed an increase in the membrane expression of CD11b (Figure 3), whereas there was no change in TLR2 and TLR4 membrane expression (data not shown).

Complete analysis of the molecules responsible for the chemotactic and priming effects in the SMMs was beyond the scope of this study. We focused on IL-1 $\beta$, TNF- $\alpha$, and IL-8, the most important cytokines in gouty inflammation. As shown in Figure 4, MSU crystals stimulated production of IL-1 $\beta$, TNF- $\alpha$, and IL-8 by Mos. Preincubating SMMs with anti-IL- 8 plus anti$\mathrm{IL}-1 \beta$ mAbs significantly reduced, but did not abolish, their chemotactic properties (62\% inhibition); as shown in Figure 1,
Figure 2

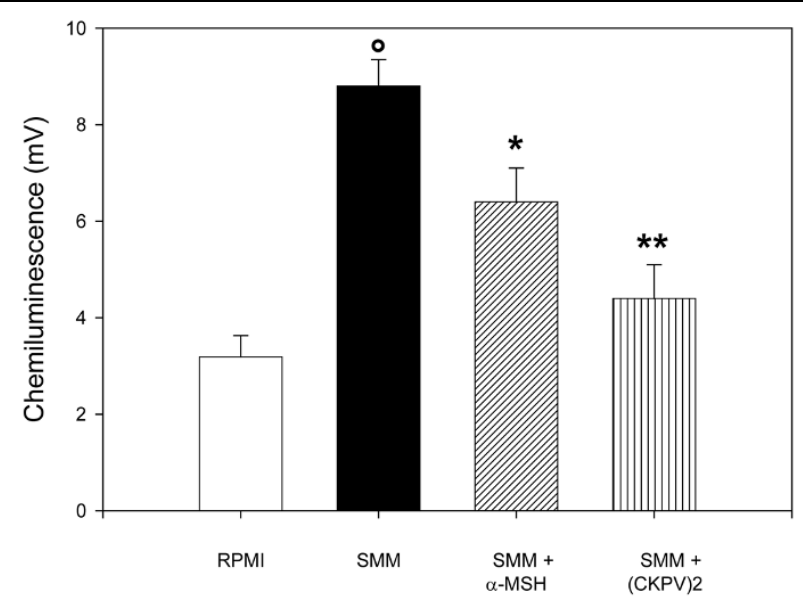

Neutrophil pre-treatment

Priming activity of supernatants from monosodium urate (MSU) crystalstimulated monocytes (SMMs) on chemiluminescence $(\mathrm{CL})$ production by neutrophils. Monocytes were incubated for 18 hours with MSU crystals $(1 \mathrm{mg} / \mathrm{mL})$ with or without alpha-melanocyte-stimulating hormone $(\alpha-\mathrm{MSH})\left(10^{-6} \mathrm{M}\right)$ or $(\mathrm{CKPV})_{2}\left(10^{-6} \mathrm{M}\right)$. The cell-free supernatants were used to pretreat purified neutrophils $\left(30\right.$ minutes at $\left.37^{\circ} \mathrm{C}\right)$ before analysis of their $\mathrm{CL}$ production in response to $\mathrm{MSU}(3 \mathrm{mg} / \mathrm{mL})$. Results are expressed as peak $C L$ values in millivolts. Bars denote mean \pm standard error of the mean. ${ }^{\circ} P<0.001$ versus RPMl; ${ }^{\star} P<0.05$ and ${ }^{* \star} P<0.01$ versus SMMs.

Figure 3

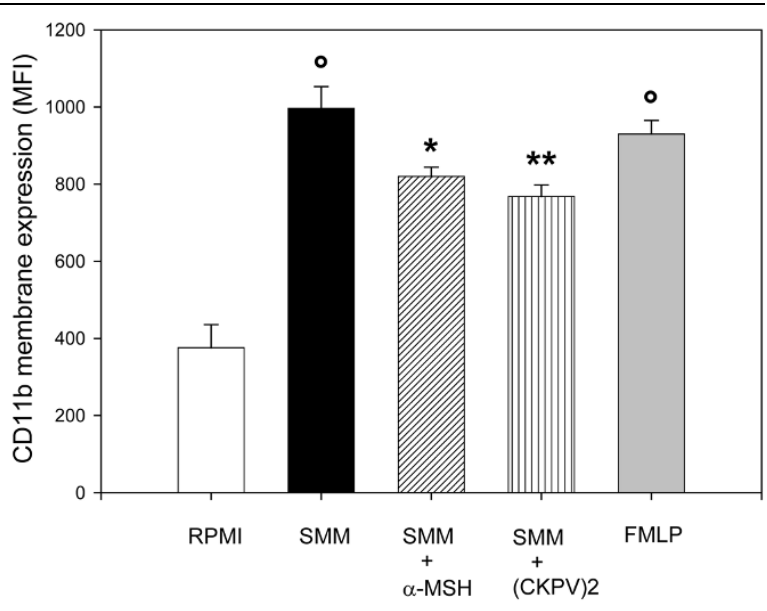

Neutrophil pre-treatment

Activity of supernatants from monosodium urate (MSU) crystal-stimulated monocytes (SMMs) on CD11b membrane expression on human neutrophils. Monocytes were incubated for 18 hours with MSU crystals $(1 \mathrm{mg} / \mathrm{mL})$ with or without alpha-melanocyte-stimulating hormone $(\alpha-\mathrm{MSH})\left(10^{-6} \mathrm{M}\right)$ or $(\mathrm{CKPV})_{2}\left(10^{-6} \mathrm{M}\right)$. Purified neutrophils were pretreated with the different SMMs or $N$-formyl-methionyl-leucyl-phenylalanine (FMLP) $\left(30\right.$ minutes at $\left.37^{\circ} \mathrm{C}\right)$ before staining with the phycoerythrin-conjugated anti-CD11b monoclonal antibody. The results are expressed as mean fluorescence intensity (MFI) \pm standard error of the mean, corrected for non-specific staining, from five separate experiments. ${ }^{\circ} P<0.001$ versus RPMI; ${ }^{\star} P<0.05$ and ${ }^{\star \star} P<0.01$ versus SMMs. 

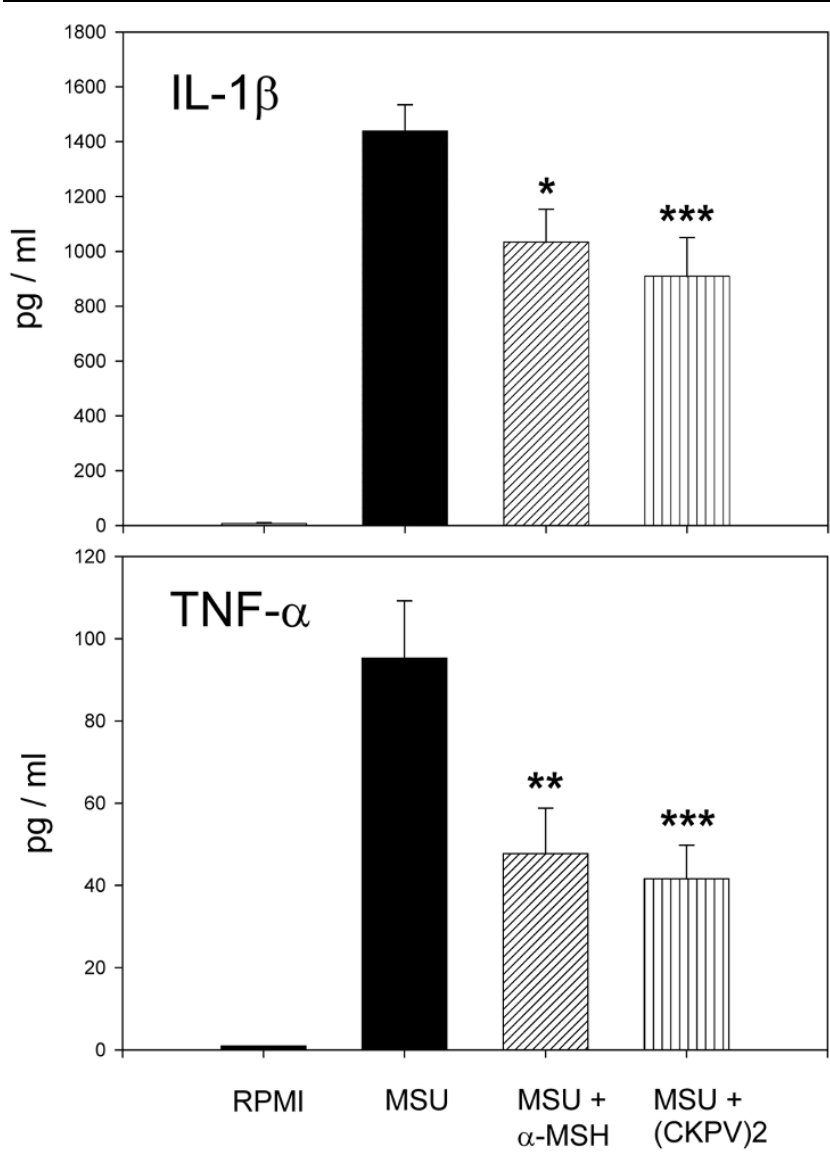

STIMULI
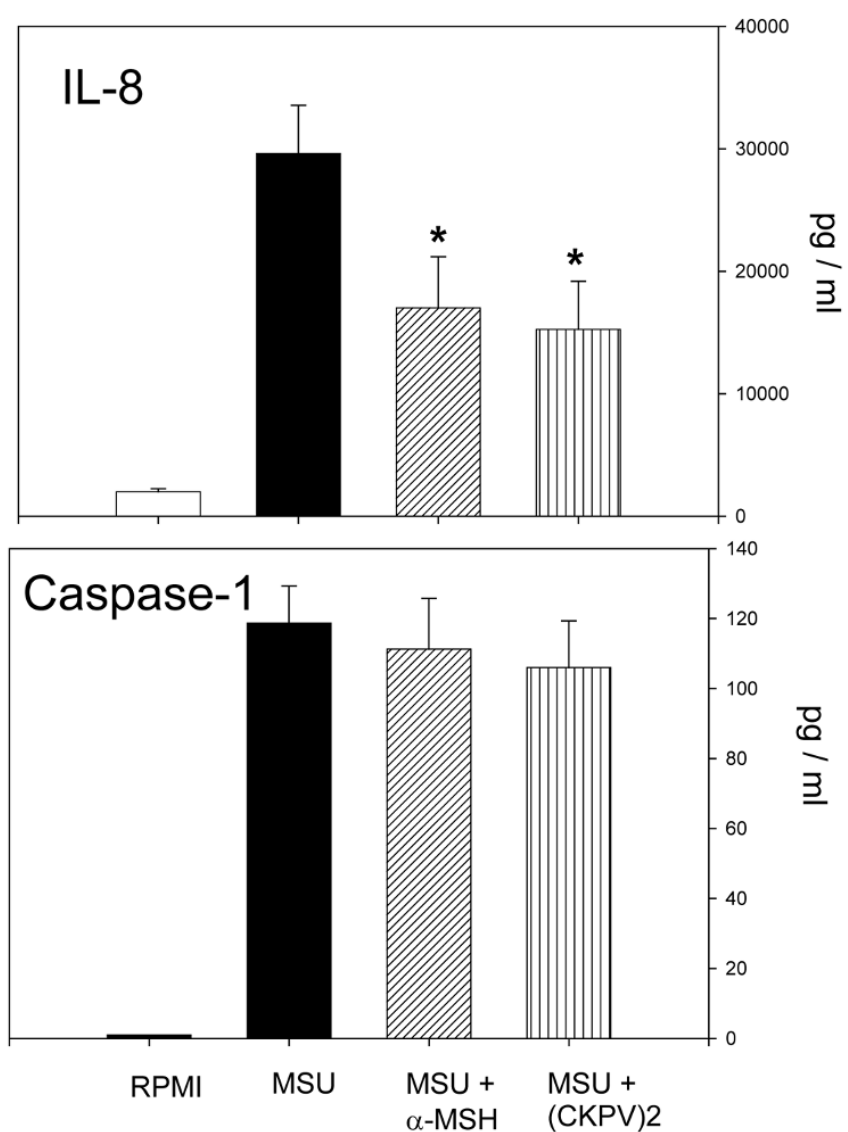

STIMULI

Effects of alpha-melanocyte-stimulating hormone $(\alpha-\mathrm{MSH})$ and $(\mathrm{CKPV})_{2}$ on inflammatory mediator production by monosodium urate (MSU)-stimulated human monocytes. Monocytes were incubated for 18 hours with MSU crystals $(1 \mathrm{mg} / \mathrm{mL})$ with or without $\alpha-\mathrm{MSH}\left(10^{-6} \mathrm{M}\right)$ or $(\mathrm{CKPV})_{2}\left(10^{-6} \mathrm{M}\right)$. Cytokine and caspase- 1 protein concentrations were measured in the cell-free supernatants. Bars denote mean \pm standard error of the mean. ${ }^{*} P<$ $0.05 ;{ }^{\star \star} P<0.02 ;{ }^{\star \star \star} P<0.01$ versus MSU. IL, interleukin; TNF- $\alpha$, tumor necrosis factor-alpha.

this inhibition was induced mainly by anti-IL-8 mAbs (43\% inhibition with anti-IL-8 and 18\% inhibition with anti-IL-1 $\beta$ mAb).

In three separate experiments, IL-1 $\beta$ and IL-8 levels were measured in supernatants from cultured Mos stimulated with MSU in the presence of the caspase-1 inhibitor z-YVAD-fmk. As shown in Figure 5, z-YVAD-fmk completely blocked MSUinduced IL-1 $\beta$ production (95\% inhibition) whereas IL-8 secretion was only partially inhibited (23\% inhibition).

\section{Melanocortin peptides reduce the production of neutrophil-activating mediators by monosodium urate- stimulated monocytes}

The SMMs obtained in the presence of $\alpha-\mathrm{MSH}$ or $(\mathrm{CKPV})_{2}$ showed significant drops in their chemotactic capacity (Figure 1) and priming activity. CL production (Figure 2) and CD11b membrane expression (Figure 3) were significantly lower than in supernatants obtained without the peptides. The capacity of
Mos to produce proinflammatory cytokines in response to MSU was reduced by $\alpha-\mathrm{MSH}$ and $(\mathrm{CKPV})_{2}$, whereas the secretion of caspase-1, the enzyme responsible for converting pre-IL-1 $\beta$ to the active form of the cytokine, was not affected (Figure 4, bottom right). $\alpha-\mathrm{MSH}$ did not further affect the inhibitory activity of z-YVAD-fmk on IL-1 $\beta$ production by MSU-stimulated Mos but did induce further moderate inhibition of IL-8 production (30\%) (Figure 5).

\section{Melanocortin peptides inhibit neutrophil responses to activating mediators produced by monosodium urate- stimulated monocytes}

In parallel experiments, we examined the activity of the melanocortin peptides on neutrophil responses to the SMMs. Neutrophils were preincubated overnight with $\alpha-\mathrm{MSH}$ or $(\mathrm{CKPV})_{2}$ before the functional assays. As shown in Figure 6, this pretreatment significantly inhibited the neutrophils' ability to migrate toward the SMMs whereas there was no effect on 
Figure 5
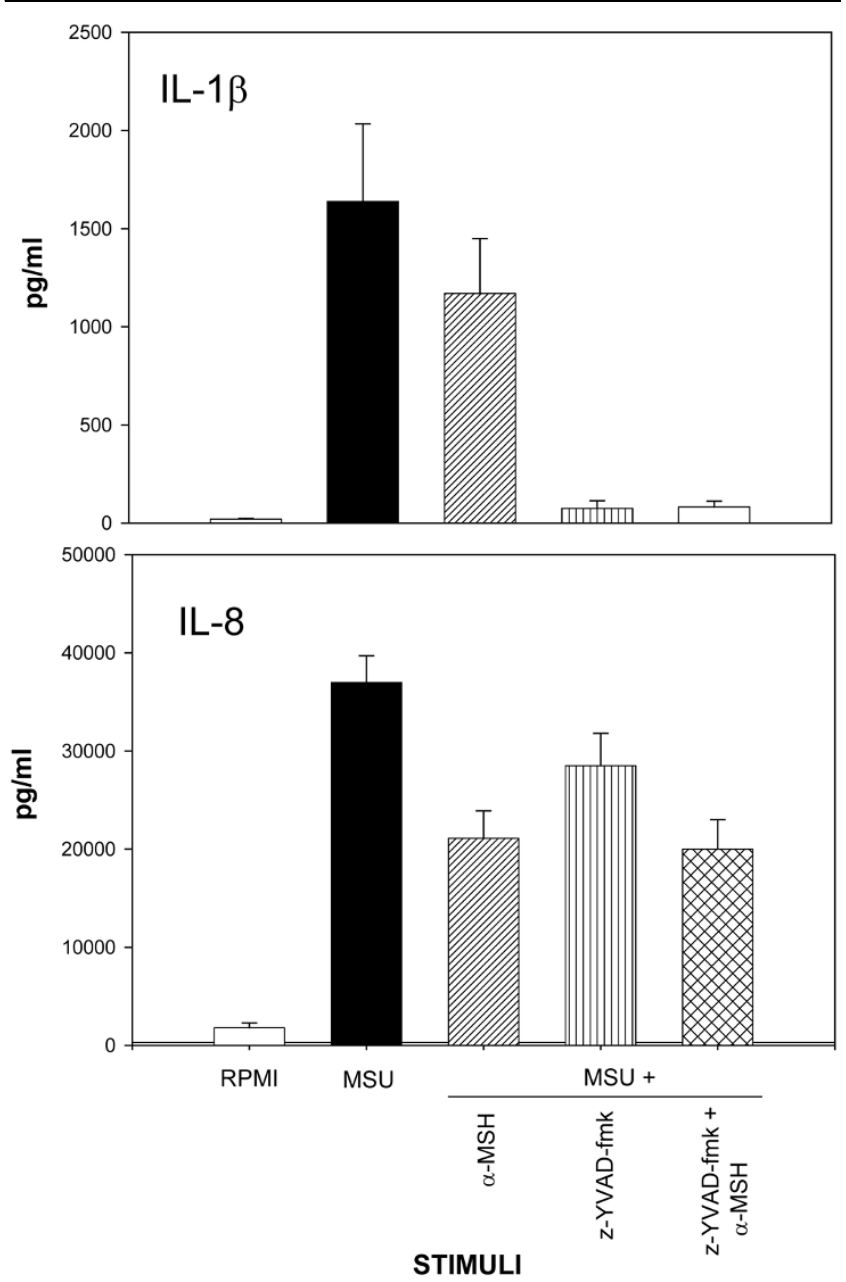

Effect of the caspase-1 inhibitor z-YVAD-fmk on inflammatory mediator production by monosodium urate (MSU)-stimulated human monocytes. Monocytes were incubated for 18 hours with MSU crystals $(1 \mathrm{mg} / \mathrm{mL})$ with or without z-YVAD-fmk $(10 \mathrm{mM})$ or alpha-melanocyte-stimulating hormone $(\alpha-\mathrm{MSH})\left(10^{-6} \mathrm{M}\right)$ or the two together. Interleukin (IL)-1 $\beta$ and $\mathrm{IL}-8$ concentrations were measured in the cell-free supernatants.

Means \pm standard errors of the mean of three separate experiments are presented.

MSU crystal-induced ROI production (data not shown). However, the overnight pretreatment made the cells less capable of being primed by the SMMs in terms of ROI production (Figure 7).

\section{Discussion}

SMMs exert chemoattractant and priming activity on neutrophils, but SMMs obtained in the presence of the melanocortin peptides had significantly less chemoattractant activity for neutrophils and less ability to prime neutrophils for CD $11 b$ membrane expression and the oxidative burst in response to MSU crystals. Stimulation of Mos with MSU crystals induces the production of proinflammatory and chemotactic sub-
Figure 6

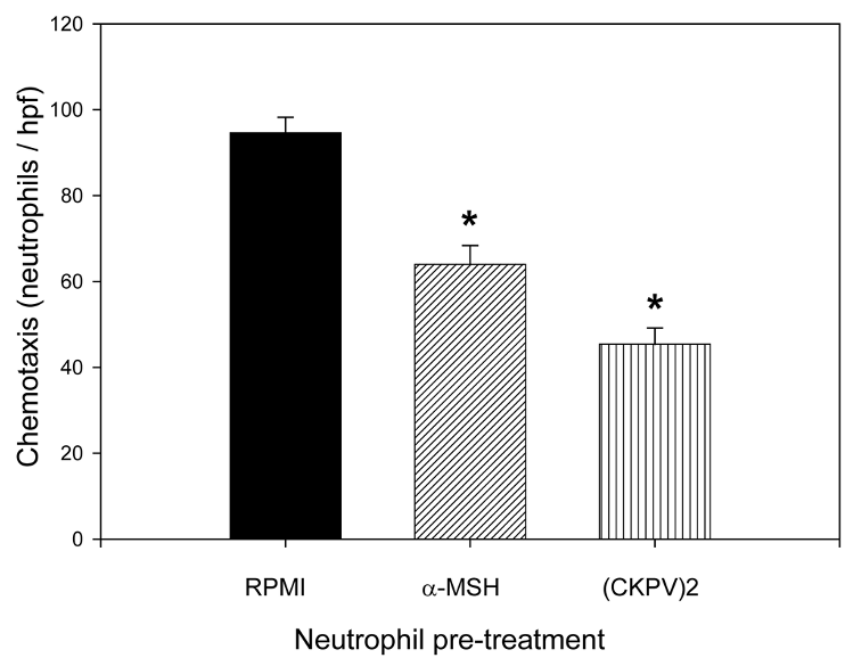

Effect of alpha-melanocyte-stimulating hormone $(\alpha-\mathrm{MSH})$ and $(\mathrm{CKPV})_{2}$ on neutrophil chemotaxis induced by supernatants from monosodium urate-stimulated monocytes. Neutrophils were pretreated with or without the peptides $\left(10^{-6} \mathrm{M}\right)$ for 18 hours before functional assays. Results are shown as number of cells per high-power field (hpf). Means \pm standard errors of the mean of five separate experiments are presented. ${ }^{\star} P<0.01$ versus RPMI.

stances [23-27]. Our interest was focused on IL-1, IL-8, and TNF- $\alpha$, the cytokines primarily involved in MSU crystal-mediated inflammation. In the presence of the melanocortin peptides, MSU crystal-stimulated Mos produced lower concentrations of these cytokines. Inhibition of these key mediators is probably at least partly responsible for the lower chemotactic and activating properties of the supernatants. Indeed, anti-IL-1 $\beta$ and anti-IL-8 mAbs showed similar inhibitory action.

The present experiments do not clarify the mechanism of the melanocortin peptides' inhibitory effect on Mos' ability to produce chemotactic and activating substances in response to MSU. Recent observations indicate that MSU crystals may act as danger signals that can activate caspase- 1 in an NALP-3 inflammosome-dependent manner with production and release of active IL-1 $\beta$ [13]. In this model, the second phase of the inflammatory response is driven by $\mathrm{LL}-1 \beta / \mathrm{IL}-1 \mathrm{R}$ signaling and MyD88-dependent NF- $\kappa B$ activation [14]. Consequently, inflammatory mediators that elicit neutrophil recruitment characteristic of acute gouty inflammation are produced.

In line with previous research [13], the present data indicate the production and secretion of caspase- 1 by MSU crystalstimulated Mos. Although melanocortin markedly reduced the release of proinflammatory cytokines, the release of caspase1 was unaffected. This suggests that melanocortin peptides do not influence the inflammosome-dependent phase of MSU crystal stimulation. This is borne out by the observation that $\alpha$ - 
Figure 7

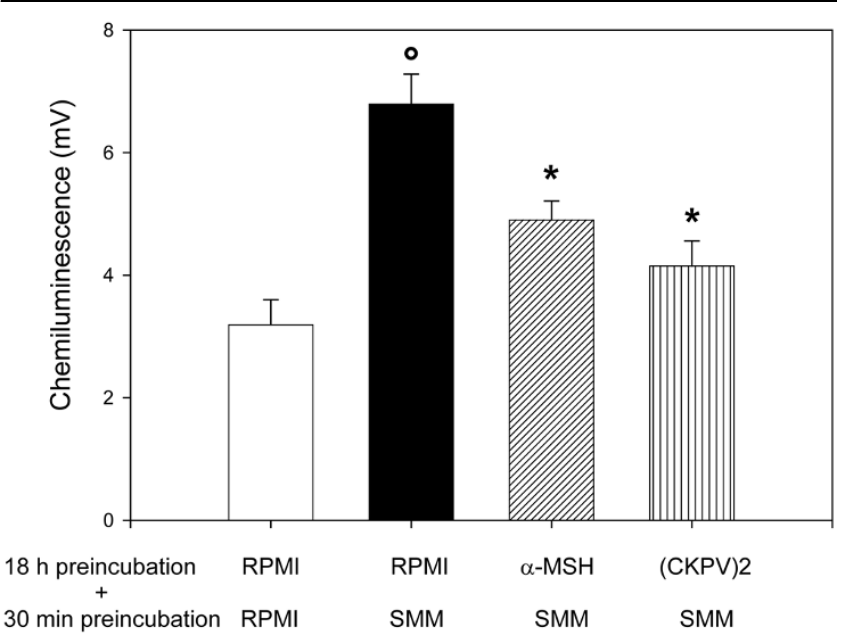

Neutrophil pre-treatment

Effects of alpha-melanocyte-stimulating hormone $(\alpha-\mathrm{MSH})$ and $(\mathrm{CKPV})_{2}$ on the priming activity of supernatants from monosodium urate-stimulated monocytes (SMMs) on chemiluminescence $(\mathrm{CL})$ production by neutrophils. Neutrophils were pretreated with or without the peptides $\left(10^{-6} \mathrm{M}\right)$ for 18 hours and then were incubated with RPMI or $\mathrm{SMMs}$ for 30 minutes, and $\mathrm{CL}$ production in response to monosodium urate $(3 \mathrm{mg} / \mathrm{mL})$ was measured. Results are expressed as peak $\mathrm{CL}$ values in millivolts. ${ }^{\circ} P<0.01$ versus RPMl+RPMl; ${ }^{*} P<0.01$ versus RPMI+SMMs.

MSH still had inhibitory activity on IL-8 secretion by MSU-stimulated Mos in the presence of the caspase-1 inhibitor z-YVADfmk.

Two molecular mechanisms by which melanocortin peptides produce their anti-inflammatory effects might be relevant in this experimental model of MSU-induced phagocyte stimulation. First, the peptides prevent activation of NF- $\kappa \mathrm{B}$ by a variety of inflammatory stimuli (reviewed in [4]). Therefore, they may inhibit MSU crystal-induced secretion of chemoattractants and activating substances by inhibiting the second phase of the inflammatory response mediated by NF- $\kappa B$ activation. Second, $\alpha-\mathrm{MSH}$ and the tripeptide KPV potently and selectively reduce membrane binding of $\mathrm{IL}-1 \beta$ to T-cell clones $[28,29]$. This mechanism could be important in our experimental model as the IL-1 $1 \beta / I L-1 R$ interaction is vital for MSU crystal-induced inflammation [14].

Preincubation of neutrophils with $\alpha-\mathrm{MSH}$ or $(\mathrm{CKPV})_{2}$ reduced their ability to migrate toward MSU crystal-induced supernatants and to be primed by SMMs, in terms of ROI production. This agrees with previous observations [9] that melanocortin peptides inhibit neutrophil chemotaxis toward FMLP and IL-8 and their capacity to generate ROls in response to phorbol esters, an effect that is probably related to the peptides' ability to increase cAMP generation in human neutrophils.

\section{Conclusions}

The present experiments indicate that $\alpha-\mathrm{MSH}$ and (CKPV) have a dual effect on MSU crystal-induced inflammation: they prevent Mos from producing neutrophil chemoattractants and activating compounds and inhibit neutrophil responses to these inflammatory substances. These findings agree with the observations of Getting and colleagues [15,16], who found that melanocortins had protective action in a rat model of gouty arthritis, and suggest a further mechanism for the protective effect. Our results indicate $\alpha-\mathrm{MSH}$ and related peptides as a potential new class of drugs for the treatment of inflammatory arthritis.

\section{Competing interests}

The authors declare that they have no competing interests.

\section{Authors' contributions}

FC conceived the study, participated in conducting monocyte and neutrophil functional assays, and drafted the manuscript. AMO conducted all of the experimental assays. ER conducted the immunofluorescence assays. AC participated in study design and helped to write the manuscript. All authors read and approved the final manuscript.

\section{Acknowledgements}

This work was supported by the following research funds: 'Ricerca Corrente 2008', Istituto Ortopedico Galeazzi, Istituto Di Ricovero e Cura a Carattere Scientifico, Milan, Italy, and PUR 2008, University of Milan, Italy.

\section{References}

1. Catania A: The melanocortin system in leukocyte biology. J Leukoc Biol 2007, 81:383-392.

2. Getting SJ: Targeting melanocortin receptors as potential novel therapeutics. Pharmacol Ther 2006, 111:1-15.

3. Luger TA, Brzoska T: alpha-MSH related peptides: a new class of anti-inflammatory and immunomodulating drugs. Ann Rheum Dis 2007, 66(Suppl 3):iii52-5.

4. Brzoska T, Luger TA, Maaser C, Abels C, Böhm M: Alphamelanocyte stimulating hormone and related tripeptides: biochemistry, antiinflammatory and protective effects in vitro and in vivo, and future perspectives for the treatment of immunemediated inflammatory diseases. Endocr Rev 2008, 29:581-602.

5. Sarkar A, Sreenivasan Y, Manna SK: Melanocyte-stimulating hormone inhibits lipopolysaccharide-induced biological responses by downregulating CD14 from macrophages. FEBS Lett 2003, 553:286-294.

6. Oktar BK, Yuksel M, Alican I: The role of cyclooxygenase inhibition in the effect of $\alpha$-melanocyte-stimulating hormone on reactive oxygen species production by rat peritoneal neutrophils. Prostaglandins Leukot Essent Fatty Acids 2004, 71:1-5.

7. Catania A, Rajora N, Capsoni F, Minonzio F, Star RA, Lipton JM: The neuropeptide $\alpha$-MSH has specific receptors on neutrophils and reduces chemotaxis in vitro. Peptides 1996, 17:675-679.

8. Manna SK, Sarkar A, Sreenivasan $Y$ : $\alpha$-Melanocyte-stimulating hormone down-regulates $\mathrm{CXC}$ receptors through activation of neutrophil elastase. Eur J Immunol 2006, 36:754-769.

9. Capsoni F, Ongari A, Colombo G, Turcatti F, Catania A: The synthetic melanocortin (CKPV) 2 exerts broad anti-inflammatory effects in human neutrophils. Peptides 2007, 28:2016-2022.

10. Cooper A, Robinson SJ, Pickard C, Jackson CL, Friedmann PS, Healy E: $\alpha$-Melanocyte-stimulating hormone suppresses anti- 
gen-induced lymphocyte proliferation in humans independently of melanocortin 1 receptor gene status. J Immuno/ 2005, 175:4806-4813.

11. Haskell-Luevano C, Sawyer TK, Hendrata S, North C, Panahinia L, Stum M, Staples DJ, Castrucci AM, Hadley MF, Hruby VJ: Truncation studies of $\alpha$-melanotropin peptides identify tripeptide analogues exhibiting prolonged agonist bioactivity. Peptides 1996, 17:995-1002

12. Gatti $S$, Carlin A, Sordi A, Leonardi P, Colombo G, Fassati LR, Lipton JM, Catania A: Inhibitory effects of the peptide (CKPV) ${ }_{2}$ on endotoxin-induced host reactions. J Surg Res 2006, 131:209-214.

13. Martinon F, Petrilli V, Mayor A, Tardivel A, Tschopp J: Gout-associated uric acid crystals activate the NALP3 inflammasome. Nature 2006, 440:237-241.

14. Chen CJ, Shi Y, Hearn A, Fitzgerald K, Golenbock D, Reed G, Akira S, Rock KL: MyD88-dependent IL-1 receptor signaling is essential for gout inflammation stimulated by monosodium urate crystals. J Clin Invest 2006, 116:2262-2271.

15. Getting SJ, Christian HC, Flower RJ, Perretti M: Activation of melanocortin type 3 receptor as a molecular mechanism for adrenocorticotropic hormone efficacy in gouty arthritis. Arthritis Rheum 2002, 46:2765-2775.

16. Getting SJ, Lam CW, Chen AS, Grieco P, Perretti M: Melanocortin 3 receptors control crystal-induced inflammation. FASEB $J$ 2006, 20:2234-2241.

17. Murakami $\mathrm{Y}$, Akahoshi T, Hayashi I, Endo H, Kawai S, Inoue M, Kondo $\mathrm{H}$, Kitasato $\mathrm{H}$ : Induction of triggering receptor expressed on myeloid cells in murine resident peritoneal macrophages by monosodium urate monohydrate crystals. Arthritis Rheum 2006, 54:455-462.

18. Boyum A: Isolation of mononuclear cells and granulocytes from human blood. Scand J Clin Lab Invest 1968, 97:77-89.

19. Boyum A: Isolation of human monocytes with Nycodenz a new non-ionic iodinated gradient medium. Scand J Immunol 1983, 17:429-436.

20. Landis RC, Yagnik DR, Florey O, Philippidis $\mathrm{P}$, Emons V, Mason JC, Haskard DO: Safe disposal of inflammatory monosodium urate monohydrate crystals by differentiated macrophages. Arthritis Rheum 2002, 46:3026-3033.

21. Scott $\mathrm{P}, \mathrm{Ma} \mathrm{H}$, Viriyakosol $\mathrm{S}$, Terkeltaub $\mathrm{S}$, Liu-Bryan R: Engagement of CD14 mediates the inflammatory potential of monosodium urate crystals. J Immunol 2006, 177:6370-6378.

22. Boyden S: The chemotactic effect of mixtures of antibody and antigen on polymorphonuclear leucocytes. J Exp Med 1962, 115:453-66.

23. Desaulniers P, Marois S, Pare G, Popa-Nita O, Gilbert C, Naccache $\mathrm{PH}$ : Characterization of an activation factor released from human neutrophils after stimulation by triclinic monosodium urate crystals. J Rheumato/ 2006, 33:928-938.

24. Matsukawa A, Yoshimura T, Maeda T, Takahashi T, Ohkawara S, Yoshinaga M: Analysis of the cytokine network among tumor necrosis factor $\alpha$, interleukin-1 $\beta$, interleukin-8, and interleukin1 receptor antagonist in monosodium urate crystal-induced rabbit arthritis. Lab Invest 1998, 78:559-569.

25. Serhan CN, Lundberg U, Weissmann G, Samuelsson B: Formation of leukotrienes and hydroxy acids by human neutrophils and platelets exposed to monosodium urate. Prostaglandins 1984, 27:563-581.

26. Hachicha M, Naccache PH, McColl SR: Inflammatory microcrystals differentially regulate the secretion of macrophage inflammatory protein 1 and interleukin 8 by human neutrophils: a possible mechanism of neutrophil recruitment to sites of inflammation in synovitis. J Exp Med 1995, 182:2019-2025.

27. Spilberg I, Bhatt AK: Crystal-induced chemotactic factor. Methods Enzymol 1988, 162:193-197.

28. Mugridge KG, Perretti M, Ghiara P, Parente L: Alpha-melanocytestimulating hormone reduces interleukin-1 beta effects on rat stomach preparations possibly through interference with a type I receptor. Eur J Pharmacol 1991, 197:151-155.

29. Poole S, Bristow AF, Lorenzetti BB, Das RE, Smith TW, Ferreira $\mathrm{SH}$ : Peripheral analgesic activities of peptides related to alpha-melanocyte stimulating hormone and interleukin-1 beta 193-195. Br J Pharmacol 1992, 106:489-492. 